\title{
Open clusters or their remnants: $B$ and $V$ photometry of NGC 1901 and NGC 1252
}

\author{
D. B. Pavani ${ }^{1}$, E. Bica ${ }^{1}$, C. M. Dutra ${ }^{1}$, H. Dottori ${ }^{1}$, B. X. Santiago ${ }^{1}$, G. Carranza $^{2}$, and R. J. Díaz ${ }^{2}$ \\ 1 Universidade Federal do Rio Grande do Sul, IF, CP 15051, Porto Alegre 91501-970, RS, Brazil \\ 2 Observatorio Astronómico de Córdoba, Laprida 854, 5000, Córdoba, Argentina
}

Received 23 January 2001 / Accepted 21 May 2001

\begin{abstract}
Photometry in the $B$ and $V$ bands is presented for the southern stellar groups NGC 1901 and NGC 1252. NGC 1901 is often described as an open cluster while NGC 1252 consists of a concentration of about 20 stars centered $\approx 20^{\prime}$ north of the original New General Catalogue coordinates, and at the southwest edge of the large region previously assigned to this object in the literature. NGC 1901 has a clear main sequence and shares similarities with the Hyades. We derive a reddening value $E(B-V)=0.04$, a distance from the Sun $d_{\odot}=0.45 \mathrm{kpc}$ $(Z=-0.23 \mathrm{kpc})$ and an age $0.6 \pm 0.1 \mathrm{Gyr}$. NGC 1901 is conclusively a physical system, dynamically comparable to or more evolved than the Hyades. The colour-magnitude diagram of NGC 1252 suggests a turnoff and main sequence, and a total of 12 probable members. We simulated the Galactic field colour-magnitude diagram in the same direction and found it to be a poor match to NGC 1252, suggesting that NGC 1252 is not a field fluctuation. Isochrone fitting to the probable members is consistent with $E(B-V)=0.02, d_{\odot}=0.64 \mathrm{kpc}(Z=-0.46 \mathrm{kpc})$ and an age $3 \pm 1$ Gyr. NGC 1252 cannot be ruled out as a physical group with the available data. If so, evidence is found that it is not a classical open cluster, but rather an open cluster remnant.
\end{abstract}

Key words. galaxy: open clusters and stellar associations - galaxies: individual: NGC 1901, NGC 1252

\section{Introduction}

Open clusters dynamically evolve and eventually dissolve. They appear to behave as weakly bound or slightly unbound systems. Catalogues include many poorly populated and/or low density stellar concentrations which may have different natures such as cluster remnants, parts of associations, quasi-associations and multiple star systems, or non-physical effects such as field fluctuations. Lodén (1977) presented a list of relatively loose young concentrations that probably have never been bound. The Basel objects (e.g. Hassan 1974) are concentrations which appear to be physical stellar groups, in contrast to concentrations produced by random fluctuations in fields of Milky Way stars (e.g. Ruprecht 46-Carraro \& Patat 1995).

Wielen (1971) studied the evolution and dissolution time scale of open clusters. Recently, numerical simulations suggested that many concentrations may be open cluster remnants (e.g. de la Fuente Marcos 1998). Bassino et al. (2000) find evidence of a possible remnant - M 73 (NGC 6994), but Carraro (2000) does not favour it. Bica et al. (2001) presented 34 dissolving star cluster candidates which are located at relatively high galactic latitudes $\left(|b|>15^{\circ}\right)$ and are underpopulated with respect to

Send offprint requests to: D. B. Pavani, e-mail: dpavani@if .ufrgs.br usual open clusters, but they possess a significant number density contrast as compared to the Galactic field. The distinction between an open cluster and a remnant is not obvious both observationally and theoretically. An open cluster remnant can be defined as a poorly populated concentration of stars as a result of the dynamical evolution of an initially more massive physical system. A widely accepted open cluster such as the Hyades appears to have lost $90 \%$ of the original stellar content (Weidemann et al. 1992). At the other extreme a binary star, the ultimate remains of a dissolved open cluster, is a cluster remnant. In the present study a cluster remnant is defined as a poorly populated physical concentration of stars with enough members to show evolutionary sequences in a colour-magnitude diagram (CMD).

NGC 1252 was discussed in Bica et al. (2001), who refer to a stellar concentration at the southwest edge of Bouchet \& Thé's (1983) definition of NGC 1252, which is a sparse collection of stars in a much larger field. NGC 1252 is described in the NGC as a star cluster containing 18 or 20 stars. It is located in Horologium with original coordinates (available e.g. in Sulentic \& Tifft 1973) corresponding to $\mathrm{J} 2000 \alpha=3^{\mathrm{h}} 10^{\mathrm{m}} 31^{\mathrm{s}}, \delta=-58^{\circ} 08^{\prime} 22^{\prime \prime}$ $(l=274.59, b=-50.64)$. All $\alpha, \delta$ values in the present study will refer to the epoch J2000.0. Bouchet \& Thé (1983, hereafter BT) observed 38 stars with UBVRI 
photometry in a $1^{\circ}$ diameter region centered on the bright carbon star TW Horologii $\left(\alpha=3^{\mathrm{h}} 12^{\mathrm{m}} 33.2^{\mathrm{s}}\right.$, $\delta=-57^{\circ} 19^{\prime} 18^{\prime \prime}$ and $\left.l=273.30, b=-50.90\right)$. They considered 16 as probable members, including TW Hor (BT 38) itself, and estimated a distance from the Sun $d_{\odot}=470$ pc. Using CZC proper motions Eggen (1984) concluded that BT's cluster was non-existent and that TW Hor $\left(d_{\odot}=400 \mathrm{pc}\right)$ probably belonged to the Hyades Supercluster. Using proper motions contained in the ACT and Hipparcos catalogues Baumgardt (1998) also concluded that the cluster as interpreted by BT is nonexistent.

CCD photometry is obtained for NGC 1252 as in Bica et al. (2001) at $\alpha=3^{\mathrm{h}} 10^{\mathrm{m}} 49^{\mathrm{s}}, \delta=-57^{\circ} 46^{\prime} 00^{\prime \prime} \quad(l=$ $\left.274^{\circ} .08, b=-50^{\circ} .83\right)$. This concentration can be seen in Fig. 1 of BT as the small group encompassed by the bright stars BT 1 and 13. An XDSS (second generation Digitized Sky Survey) blow-up of the concentration was shown in Fig. 4 of Bica et al. (2001). We emphasize that the present set of stars related to the concentration is not BT's set of members.

We also address the crucial point of the distinction between an open cluster and an open cluster remnant. We deepen the photometry of the poorly populated open cluster or stellar group NGC 1901 for comparisons with NGC 1252. NGC 1901 at $\alpha=5^{\mathrm{h}} 18^{\mathrm{m}} 11^{\mathrm{s}}, \delta=-68^{\circ} 27^{\prime} 00^{\prime \prime}$ $(l=279.03, b=-33.60)$ is located in Dorado and was reported by Bok \& Bok (1960) as a loose stellar grouping. Sanduleak \& Philip (1968, hereafter SP) by means of $B V$ photometry concluded that NGC 1901 was a nearby Galactic stellar group projected onto the LMC. They found a turnoff near $\mathrm{A} 0$, a reddening value $E(B-V)=$ 0.065 and distance from the Sun $d_{\odot}=330$ pc. Murray et al. (1969) carried out astrometry for SP's stars with Cape Astrographic plates taken over more than 60 years. They found that 14 of them have proper motions in common and called the object a star cluster. However, despite the conclusion of it being a physical system there remains the doubt whether NGC 1901 is a classical open cluster or a remnant, or some transition state. In Bica et al. (2001) NGC 1901 was taken as a comparison loose cluster and the number density contrast was important. Section 2 describes observations and reductions. Section 3 discusses the comparison object NGC 1901. Section 4 discusses the properties of NGC 1252. In Sect. 5 we compare the present objects with the Hyades and discuss the possibility of remnant physical systems. Concluding remarks are given in Sect. 6.

\section{Observations and reductions}

The observations of NGC1252 and NGC1901 were acquired at the Córdoba University Bosque Alegre Astrophysical Station, Argentina, on the night 28 December 1998, using a $30 \mathrm{~cm}$ Schmidt-Cassegrain telescope of the Universidade Federal do Rio Grande do Sul, temporarily installed at the site. CCD images with the $B$ and $V$ filters were collected with a KODAK

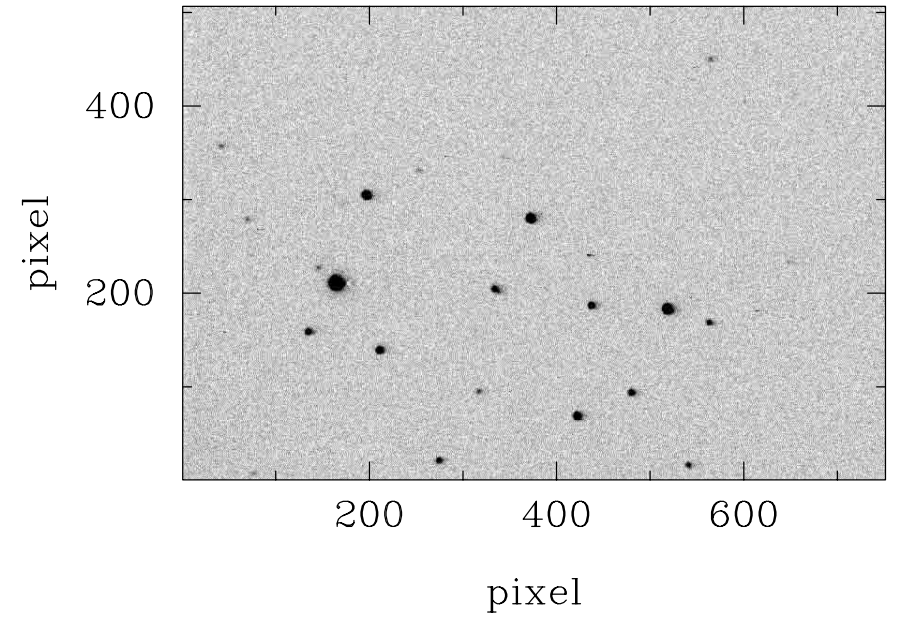

Fig. 1. V CCD image of NGC 1252 with exposure 40 s. Field is $12 ! 4 \times 8^{\prime} \cdot 3$. Northwest is to the left and northeast to the top.

KAF-0400 chip of $768 \times 512$ pixels and size $9 \mu \mathrm{m} \times 9 \mu \mathrm{m}$, corresponding to a field $12 ! 4 \times 8.3$ on the sky. We employed $3 B$ and $2 V$ frames of exposure time $20 \mathrm{~s}$, and $1 B$ frame and $1 V$ frame of $40 \mathrm{~s}$. We illustrate a CCD $V$ image of NGC 1252 in Fig. 1 which includes most of the concentration.

The reductions were carried out with IRAF starting with bias, dark, and flat field corrections. We combined the images for better S/N ratios. DAOPHOT (Stetson 1992) was used to extract stars and derive instrumental magnitudes. We show in Fig. 2 the DAOPHOT $(B-V)$ and $V$ internal errors as a function of $V$ mag for NGC 1252: $\epsilon_{V}$ and $\epsilon_{(B-V)}$ attain 0.05 for $V \approx 15$. We calibrated the data using stars from BT's photoeletric observations in the frame, since our main objective was to deepen their photometry in the stellar concentration area and check whether a significant MS could be present. All stars share the airmass $X \approx 1.19$, and previous to the standard system transformation we corrected them for this effect. We used the site average atmospheric extinction coefficients $K_{V}=0.16$ and $K_{(B-V)}=0.13$ (Minniti et al. 1989). The adopted standard stars are BT 12, 14, 15, 16, 17, 18, 19 and 27 . The resulting transformation equations to the standard system are

$V=17.83+v+1.61 \times(b-v)$

$(B-V)=-1.16+1.60 \times(b-v)$

where $v$ and $(b-v)$ are the instrumental values corrected for atmospheric extinction. The external errors were estimated as the r.m.s. of the differences betwen standard and calculated values, and we obtained $\rho_{V}=0.13$ and $\rho_{(B-V)}=0.04$.

The bright stars BT $1\left(\alpha=03^{\mathrm{h}} 10^{\mathrm{m}} 50.0^{\mathrm{s}}, \delta=-57^{\circ} 42^{\prime}\right.$ $\left.06^{\prime \prime}\right)$ and BT $11\left(\alpha=03^{\mathrm{h}} 11^{\mathrm{m}} 09.0^{\mathrm{s}}, \delta=-57^{\circ} 47^{\prime} 38^{\prime \prime}\right)$ were saturated in our frames. We adopt BT's photoeletric values for $\mathrm{BT} 1(V=8.68,(B-V)=1.03)$ and BT $11(V=10.50,(B-V)=1.05)$. For the bright star BT $13\left(\alpha=03^{\mathrm{h}} 10^{\mathrm{m}} 39.2^{\mathrm{s}}, \delta=-57^{\circ} 48^{\prime} 35.3^{\prime \prime}\right)$, located in 


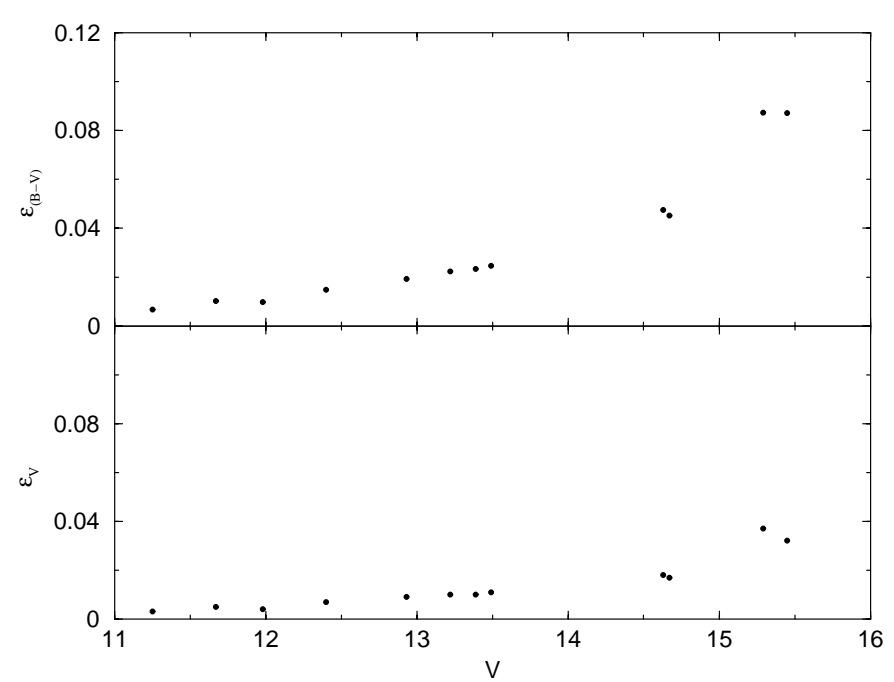

Fig. 2. NGC 1252: $(B-V)$ internal errors (upper panel) and $V$ internal errors (lower panel) as a function of $V$ magnitude.

the concentration just outside the CCD frames, we adopt BT's values $V=6.62,(B-V)=0.89$. The same applies to BT 28 at $\alpha=03^{\mathrm{h}} 10^{\mathrm{m}} 38.5^{\mathrm{s}}, \delta=-57^{\circ} 47^{\prime} 20^{\prime \prime}$ with $V=11.95$ and $(B-V)=0.47$.

We obtained CCD photometry for 12 stars in the NGC 1252 concentration. Among them 8 are also present in BT while 4 are newly observed. The results are shown in Table 1, by Col(s).: (1) identification, (2) and (3) J2000 equatorial coordinates, (4) and (5) stars with photoeletric $V$ and $(B-V)$ values from BT, (6) and (7) present CCD photometry in $V$ and $(B-V)$, and (8) membership.

For NGC 1901 we used $1 B$ and $1 V 10$ sec. frames, and $3 B$ and $3 V 30$ sec frames. The object was observed at $X \approx 1.45$, and the instrumental magnitudes were corrected accordingly. As standard stars we used SP 7, 8, 9, $10,12,13,14,15$ and 16 . The distribution of the photometric internal errors is similar to that of NGC 1252 in Fig. 2, except that $\epsilon_{(B-V)}$ attains 0.05 for $V \approx 14.5$. The external errors are $\rho_{V}=0.04$ and $\rho_{(B-V)}=0.01$. The results are in Table 2 where we also indicate the SP values for comparison purposes. The columns follow those of Table 1, except two additional ones for Tycho proper motions (Sect. 3).

\section{The stellar group NGC 1901}

We observed NGC 1901's central concentration which can be seen in the chart provided by the WEBDA database (Mermilliod 1996) in the web interface http://obswww. unige.ch/webda. It has a diameter of $\approx 19^{\prime}$. There occurs an extension $20^{\prime}$ to the NW (Sanduleak \& Philip 1968), which presents proper motions comparable to those of the central stars (Murray et al. 1969). The $V \times(B-V)$ CMD derived from the CCD photometry (30 stars, Table 2 ) is shown in Fig. 3 as the large (previous stars) and small solid circles (photometry for the first time). A gain of $\Delta V \approx 4 \mathrm{mag}$ is obtained with respect to SP's photometry. The bright stars in the CCD area de- fine a MS. The evolved star HD 35294 (TYC2 573) was not included by SP in their sample, and it was saturated in our frames. We adopted Johnson $V=8.40,(B-V)=$ 0.70 values derived from the Tycho photometry, which are accurate for such bright stars. We superimposed on the CMD the NW extension stars (upwards open triangles) using SP values, which basically follow the upper MS and turnoff (TO) of the central stars. The SP stars to the south outside the CCD area (downwards open triangles) are located along the MS. Both the NW extension and the south stars are compatible in the CMD with the bulk of central stars of NGC 1901.

NGC 1901 is projected onto the young LMC disc, and luminous LMC stars are expected. The LMC intermediate age disc (Bica et al. 1998, 1999) provides luminous AGB stars at the limit of the present photometry. The LMC RGB tip is below our limit, and the clump of red supergiants at an age $\approx 10 \mathrm{Myr}$, such as that in the LMC cluster NGC 2004 (Bencivenni et al. 1991), is far too red $(V \approx 13.2,(B-V) \approx 1.55)$ to contaminate the MS of NGC 1901. We conclude that LMC intermediate colour supergiants and some bright AGB stars are the main field contributors. This can be seen in the Shapley III fields (Dolphin \& Hunter 1998) in the young LMC disc north of the bar, likewise the LMC part onto which NGC 1901 is projected. Probable LMC members (Table 2) are located bluewards of the dashed line in Fig. 3. We cannot rule out some LMC contamination in the observed lower MS of NGC 1901.

We employed solar metallicity Padova isochrones (Girardi et al. 2000) of different ages for best fit solutions (Fig. 3) and parameters. We find a reddening value $E(B-V)=0.04$ and an apparent distance modulus $(m-M)=8.37$. The isochrones correspond to ages 0.5 , 0.6 and 0.9 Gyr, from which we estimate an age $0.6 \pm$ 0.1 Gyr for NGC 1901, thus similar to that of the Hyades (WEBDA, Weidemann et al. 1998). The 0.6 Gyr isochrone basically fits a TO distribution and HD 35294, which is thus a photometrically probable member. The latter star together with all those compatible with the $0.6 \mathrm{Gyr}$ isochrone within total colour errors $\sqrt{\epsilon^{2}+\rho^{2}}$ (Sect. 2), are indicated as probable members in Table 2. The star GCS 0916200216 is too red and was classified as a nonmember.

Using the total-to-selective extinction ratio $R_{V}=3.1$ we obtain $A_{V}=0.12$. The absolute distance modulus is $(m-M)_{\circ}=8.25 \pm 0.20$. The distance from the Sun is $d_{\odot} \approx 0.45 \pm 0.04 \mathrm{kpc}$, thus somewhat larger than that derived by SP. Assuming a solar Galactocentric distance of $8 \mathrm{kpc}$ (Reid 1993), the Galactocentric coordinates are $X=-7.87 \mathrm{kpc}, Y=-0.37 \mathrm{kpc}, Z=-0.23 \mathrm{kpc}(X<0$ is our side of the Galaxy). The Galactocentric distance is $R_{\mathrm{GC}}=7.88 \mathrm{kpc}$, thus a nearly solar circle object. It is located at $\approx 230$ pc below the Galactic plane, where younger disc components are less probable.

Astrometry in the region is significant for proper motions but not for parallaxes. In the CCD area HD 35183 (HIP 24652 or SP 7) has a parallax $p=1.22 \pm 0.87$ mas 
Table 1. CCD photometric results for NGC 1252.

\begin{tabular}{|c|c|c|c|c|c|c|c|}
\hline $\mathrm{Name}^{\mathrm{a}}$ & $\begin{array}{c}\alpha \\
\mathrm{h}: \mathrm{m}: \mathrm{s}\end{array}$ & $\begin{array}{c}\delta \\
\circ:^{\prime} \quad:^{\prime \prime}\end{array}$ & $\begin{array}{c}V \\
\mathrm{BT}\end{array}$ & $\begin{array}{c}(B-V) \\
\mathrm{BT}\end{array}$ & $\begin{array}{c}V \\
\mathrm{CCD}\end{array}$ & $\begin{array}{c}(B-V) \\
\mathrm{CCD}\end{array}$ & Membership $^{\mathrm{b}}$ \\
\hline BT 15 & $03: 11: 10.7$ & $-57: 44: 38$ & 11.47 & 1.21 & 11.25 & 1.18 & $\mathrm{lpm}$ \\
\hline BT 12 & 03:10:50.2 & $-57: 47: 11$ & 11.95 & 0.50 & 11.67 & 0.55 & $\mathrm{pm}$ \\
\hline BT 17 & 03:11:02.6 & $-57: 41: 48$ & 11.92 & 1.51 & 11.99 & 1.54 & $\mathrm{~nm}$ \\
\hline BT 18 & 03:10:45.0 & $-57: 43: 23$ & 12.49 & 0.73 & 12.40 & 0.70 & $\mathrm{pm}$ \\
\hline BT 14 & 03:11:04.4 & $-57: 46: 22$ & 12.91 & 0.60 & 12.93 & 0.63 & $\mathrm{pm}$ \\
\hline BT 27 & $03: 10: 56.5$ & $-57: 47: 48$ & 13.17 & 0.49 & 13.22 & 0.55 & $\mathrm{pm}$ \\
\hline BT 16 & 03:10:59.8 & $-57: 44: 43$ & 13.21 & 0.78 & 13.40 & 0.78 & $\mathrm{pm}$ \\
\hline BT 19 & 03:10:42.4 & $-57: 42: 06$ & 13.21 & 0.83 & 13.49 & 0.73 & $\mathrm{pm}$ \\
\hline GSC 0849800928 & 03:10:51.6 & $-57: 49: 21$ & - & - & 14.63 & 0.95 & $\mathrm{pm}$ \\
\hline GSC 0849800945 & $03: 11: 10.2$ & $-57: 48: 26$ & - & - & 14.67 & 1.10 & $\mathrm{pm}$ \\
\hline GSC 0849801321 & 03:10:51.7 & $-57: 40: 06$ & - & - & 15.29 & 0.35 & $\mathrm{~nm}$ \\
\hline GSC 0849801024 & $03: 10: 46.5$ & $-57: 45: 20$ & - & - & 15.45 & 0.75 & $\mathrm{pm}$ \\
\hline
\end{tabular}

${ }^{\mathrm{a}} \mathrm{BT}$ is from Bouchet \& Thé (1983), GSC Guide Star Catalogue; ${ }^{\mathrm{b}} \mathrm{pm}$ means probable, lpm less probable and nm non-member.

and a corresponding distance $d_{\odot}=820_{+2037}^{-342}$ pc. Within uncertainties this TO star of spectral type A2 has a distance compatible with that of NGC 1901 derived from the CMD. The other Hipparcos stars are outside the CCD area. HD 35230 (HIP 24671) has $p=3.47 \pm 0.78$ mas and $d_{\odot}=288_{+81}^{-53} \mathrm{pc}$ which is marginally compatible with that of NGC 1901, but Johnson photometry $V=7.57,(B-$ $V)=0.86$ provided in the Hipparcos catalogue, and most of all Tycho proper motions $\mu_{\alpha}=-17.4 \pm 1.1 \mathrm{mas} / \mathrm{yr}$, $\mu_{\delta}=-0.8 \pm 1.1 \mathrm{mas} / \mathrm{yr}$ indicate that it is not a member. HD 269320 (HIP 24763) with $p=8.91 \pm 1.97$ mas and $d_{\odot}=112_{+32}^{-20} \mathrm{pc}$ is in the foreground. We show in Fig. 4 a sky chart for a Tycho extraction of diameter $60^{\prime}$ centered in NGC 1901. The large circle encompasses the CCD data, where most of the stars share motions (Table 2) indicating a physical system, in agreement with Murray et al. (1969). The stars in the NW extension basically move in the same direction as the central stars. The proper motion moduli are comparable: $9.3 \pm 3.3 \mathrm{mas} / \mathrm{yr}$ for 10 stars in the central part and $12.4 \pm 9.7 \mathrm{mas} / \mathrm{yr}$ for 4 stars in the NW extension. The dispersions are not intrinsic because they are of the order of the proper motion errors. A few additional stars are compatible with the central concentration and NW extension stars, and could be related to a corona - see M 73 (Bassino et al. 2000). The age and distance of the stars in the central and NW concentrations are similar, and it remains to be studied whether the substructures are the result of the evolution of a parent initial cluster, an interaction or a close approach. In particular, projection effects of tidal arms might cause a secondary concentration, according to $N$-body simulations (Combes et al. 1999).

\section{The stellar group NGC 1252}

Figure 5 shows the $V \times(B-V)$ CMD with the 12 stars from the present photometry and total colour error bars $\sqrt{\epsilon^{2}+\rho^{2}}$ (Sect. 2). Also shown are the 4 complementary

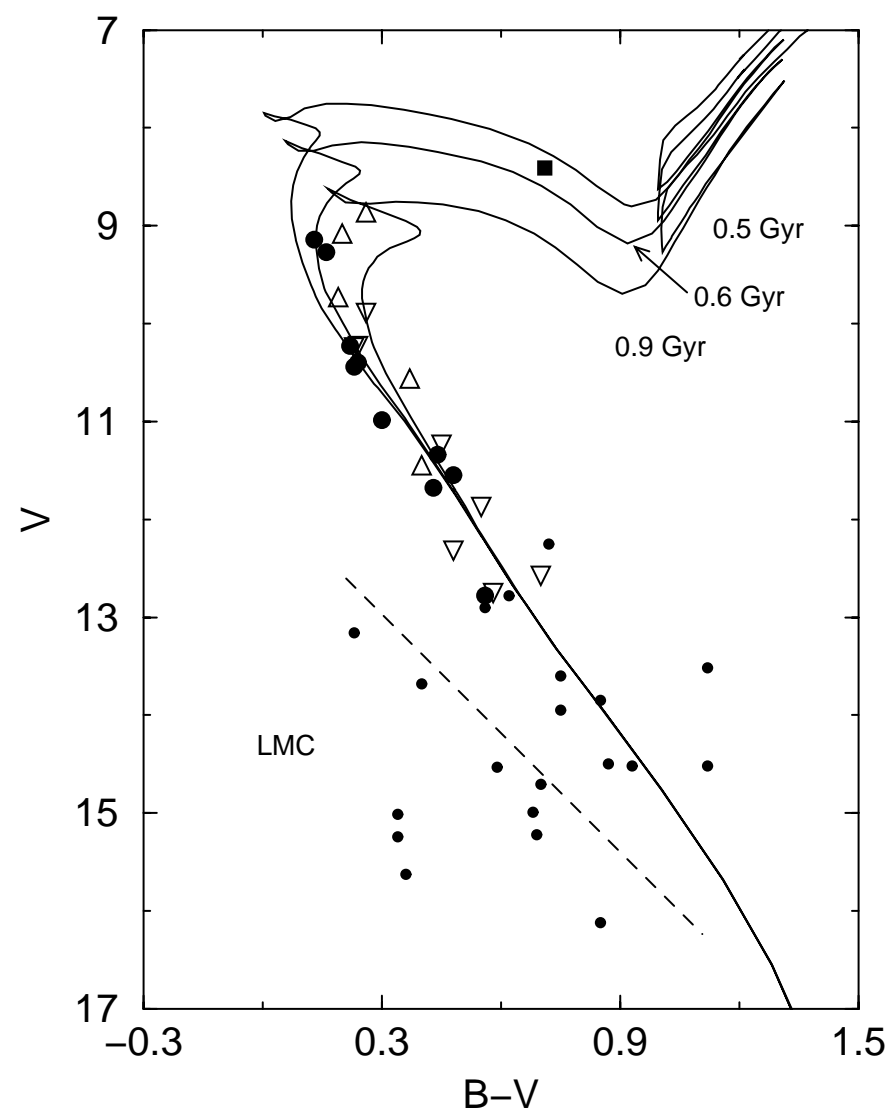

Fig. 3. NGC 1901: $V \times(B-V)$ diagram for 30 stars with CCD photometry: small solid circles are new stars and large ones are in common with SP. Filled square is HD 35294 with Tycho photometry. Open triangles are $13 \mathrm{SP}$ stars outside the CCD frames: upwards ones are in the NW extension and downwards ones are south of the CCD frames. Padova isochrone solution is shown. Dashed line separates probable LMC members.

BT stars in the area (BT 1, 11, 13 and 28). The fainter stars in the CMD were observed for the first time. The CMD suggests a MS and possibly some related giants. 
Table 2. CCD photometric results for NGC 1901.

\begin{tabular}{|c|c|c|c|c|c|c|c|c|c|}
\hline Names $^{\mathrm{a}}$ & $\begin{array}{c}\alpha \\
\mathrm{h}: \mathrm{m}: \mathrm{s}\end{array}$ & $\begin{array}{c}\delta \\
\circ:^{\prime} \quad:^{\prime \prime}\end{array}$ & $\begin{array}{c}V \\
\mathrm{SP}\end{array}$ & $\begin{array}{c}(B-V) \\
\mathrm{SP}\end{array}$ & $\begin{array}{c}V \\
\mathrm{CCD}\end{array}$ & $\begin{array}{c}(B-V) \\
\text { CCD }\end{array}$ & $\begin{array}{c}\mu_{\alpha} \\
\text { mas/yr }\end{array}$ & $\begin{array}{c}\mu_{\delta} \\
\mathrm{mas} / \mathrm{yr}\end{array}$ & Membership $^{\mathrm{b}}$ \\
\hline SP 7, TCY2 552 & $05: 17: 23.0$ & $-68: 28: 19$ & 9.16 & 0.14 & 9.14 & 0.13 & 0.0 & 10.7 & $\mathrm{pm}$ \\
\hline SP 11, TYC2 732 & 05:18:02.1 & $-68: 21: 19$ & 9.25 & 0.20 & 9.27 & 0.16 & -0.8 & 12.4 & $\mathrm{pm}$ \\
\hline SP 14, TYC2 142 & $05: 18: 22.5$ & $-68: 28: 02$ & 10.24 & 0.21 & 10.23 & 0.22 & -0.7 & 11.3 & $\mathrm{pm}$ \\
\hline SP 12, TYC2 669 & 05:18:11.9 & $-68: 25: 36$ & 10.38 & 0.24 & 10.40 & 0.24 & 4.6 & 6.8 & $\mathrm{pm}$ \\
\hline SP 10, TYC2 883 & $05: 17: 59.2$ & $-68: 31: 28$ & 10.48 & 0.25 & 10.44 & 0.23 & 3.6 & 6.6 & $\mathrm{pm}$ \\
\hline SP 9, TCY2 498 & $05: 17: 35.8$ & $-68: 21: 47$ & 10.91 & 0.31 & 10.99 & 0.30 & 5.0 & 11.1 & $\mathrm{pm}$ \\
\hline SP 13, TCY2 702 & $05: 18: 16.7$ & $-68: 25: 10$ & 11.37 & 0.45 & 11.34 & 0.44 & 5.0 & 5.5 & $\mathrm{pm}$ \\
\hline SP 15, TCY2 196 & $05: 18: 29.5$ & $-68: 27: 14$ & 11.58 & 0.49 & 11.55 & 0.48 & 3.0 & 8.9 & $\mathrm{pm}$ \\
\hline SP 16, TCY2 590 & $05: 18: 42.7$ & $-68: 27: 33$ & 11.64 & 0.41 & 11.68 & 0.43 & 1.1 & 8.3 & $\mathrm{pm}$ \\
\hline SP 8, GSC 0916201005 & $05: 17: 27.1$ & $-68: 25: 42$ & 12.56 & 0.58 & 12.78 & 0.56 & - & - & $\mathrm{pm}$ \\
\hline GSC 0916200682 & $05: 18: 41.8$ & $-68: 22: 29$ & - & - & 12.25 & 0.72 & - & - & $\mathrm{pm}$ \\
\hline GSC 0916200834 & 05:19:05.3 & $-68: 30: 46$ & - & - & 12.78 & 0.62 & - & - & $\mathrm{pm}$ \\
\hline GSC 0916200626 & 05:18:19.1 & $-68: 26: 25$ & - & - & 12.9 & 0.56 & - & - & $\mathrm{pm}$ \\
\hline GSC 0916200464 & $05: 18: 27.7$ & $-68: 31: 28$ & - & - & 13.16 & 0.23 & - & - & pLMC \\
\hline GSC 0916200216 & $05: 17: 51.2$ & $-68: 24: 34$ & - & - & 13.52 & 1.12 & - & - & $\mathrm{nm}$ \\
\hline MACS 0517684015 & $05: 17: 41.3$ & $-68: 29: 04$ & - & - & 13.60 & 0.75 & - & - & $\mathrm{pm}$ \\
\hline GSC 0916200883 & $05: 17: 59.2$ & $-68: 31: 28$ & - & - & 13.68 & 0.40 & - & - & pLMC \\
\hline 1 & 05:18:04.0 & $-68: 26: 44$ & - & - & 13.85 & 0.85 & - & - & $\mathrm{pm}$ \\
\hline USNO 015002864868 & $05: 17: 51.3$ & $-68: 26: 08$ & - & - & 13.95 & 0.75 & - & - & $\mathrm{pm}$ \\
\hline GSC 0916200619 & $05: 18: 52.7$ & $-68: 32: 35$ & - & - & 14.50 & 0.87 & - & - & $\mathrm{pm}$ \\
\hline 2 & $05: 17: 27.5$ & $-68: 29: 45$ & - & - & 14.52 & 0.93 & - & - & $\mathrm{pm}$ \\
\hline 3 & $05: 18: 36.5$ & $-68: 29: 10$ & - & - & 14.52 & 1.09 & - & - & $\mathrm{pm}$ \\
\hline USNO 01502856433 & $05: 17: 32.6$ & $-68: 23: 52$ & - & - & 14.53 & 0.59 & - & - & pLMC \\
\hline MACS 0517684021 & $05: 17: 56.5$ & $-68: 24: 22$ & - & - & 14.71 & 0.70 & - & - & pLMC \\
\hline USNO 015002855788 & $05: 17: 31.1$ & $-68: 23: 51$ & - & - & 14.99 & 0.68 & - & - & pLMC \\
\hline MACS 0518684007 & $05: 18: 11.4$ & $-68: 26: 34$ & - & - & 15.01 & 0.34 & - & - & pLMC \\
\hline USNO 015002858752 & $05: 17: 37.6$ & $-68: 31: 51$ & - & - & 15.22 & 0.69 & - & - & pLMC \\
\hline MACS 0518685008 & 05:18:33.4 & $-68: 30: 42$ & - & - & 15.24 & 0.34 & - & - & pLMC \\
\hline MACS 0518684004 & 05:18:09.4 & $-68: 26: 26$ & - & - & 15.63 & 0.36 & - & - & pLMC \\
\hline 4 & $05: 18: 58.0$ & $-68: 21: 25$ & - & - & 16.12 & 0.85 & - & - & pLMC \\
\hline
\end{tabular}

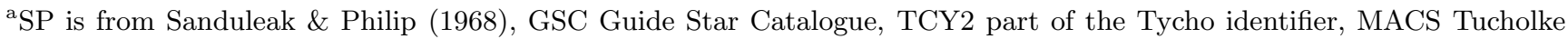
et al. (1996), USNO Monet et al. (1998); ${ }^{\mathrm{b}} \mathrm{pm}$ means probable, nm non-member, pLMC probable LMC member.

We used Padova isochrones of different ages, together with reddening constraints to find best fit solutions.

The bright giants BT 1 (K1 III/IV) and BT 13 (G8 III) have Hipparcos parallaxes $p=10.24 \pm 0.78$ mas and $p=5.48 \pm 0.54$ mas, respectively. The corresponding distances are $d_{\odot}=98 \pm 8$ pc and $d_{\odot}=182 \pm 16$ pc. Both stars together cannot belong to a possible physical stellar group. In Fig. 5 we superimpose the best isochrone fits. The reddening was estimated taking into account two methods. Schlegel et al.'s (1998) reddening maps are based on dust thermal emission and provide in this direction $E(B-V)=0.02$. Burstein \& Heiles' (1982) reddening maps are based on H I column densities and galaxy counts and indicate an absence of reddening in the area. We conclude that $E(B-V)=0.02$ is compatible with the MS for intermediate ages and an apparent distance modulus $(m-M)=9.1$. From the 2 and 4 Gyr isochrones we estimate an age $3 \pm 1$ Gyr for NGC 1252 . With $A_{V}=0.062$ and
$(m-M)_{\circ}=9.04 \pm 0.25$ we obtain $d_{\odot} \approx 0.64 \pm 0.07 \mathrm{kpc}$. The distance modulus error is by far dominated by vertical uncertainties in the CMD fit. The Galactocentric coordinates are $X=-7.82 \mathrm{kpc}, Y=-0.41 \mathrm{kpc}$ and $Z=-0.46 \mathrm{kpc}$. The Galactocentric distance is $R_{\mathrm{GC}}=$ $7.84 \mathrm{kpc}$, thus likewise NGC 1901, NGC 1252 is a nearly solar circle object. It is located at $\approx 460$ pc below the Galactic plane, consistent with the old disc distribution (Friel 1995).

The star BT 17 was classified as a non-member (Table 1) because it is too red with respect to the isochrones. BT considered the stars BT 12, 14, 15, 16, 18, 19 and 27 as Galactic field of their large region, but their probable members were around TW Hor (Sect. 1). We considered them as probable members based on their locus with respect to the isochrone MS, except BT 15 which is rather too red with respect to the subgiant branch and was classified as a less probable member. The CCD 


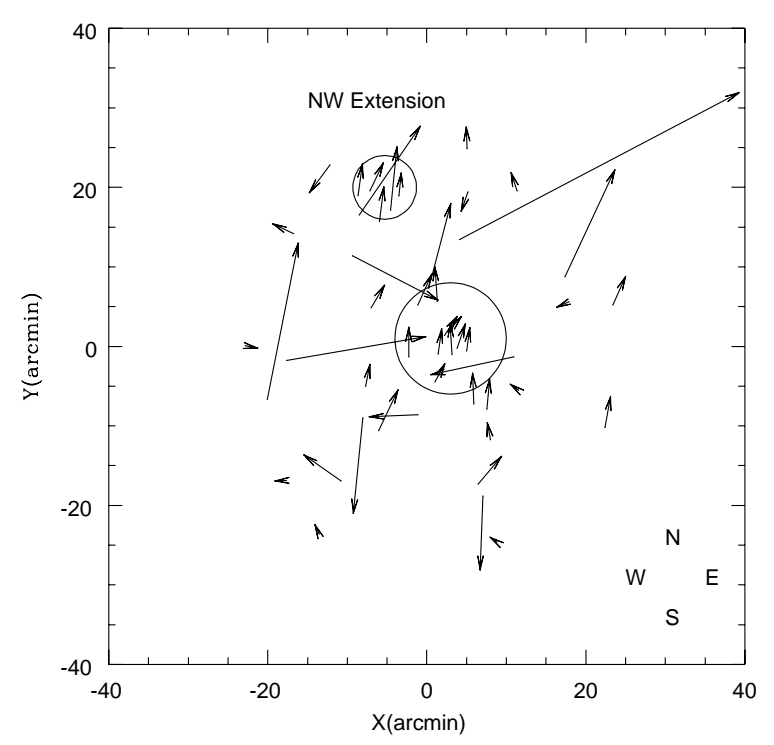

Fig. 4. NGC 1901: sky chart of Tycho stars in a region of diameter $60^{\prime}$. Arrow length is proportional to proper motion modulus, and largest one is 113.5 mas/yr. The large circle has a diameter of $12^{\prime}$ and corresponds to the CCD area.

photometry of the Guide Star Catalogue entries GSC 0849800928, 0849800945 and 0849801024 (Table 1) allowed us to classify them as probable members taking into account the colour error bars and the possibility of a double star sequence parallel to the MS. The star GSC 0849801321 is classified as a non member because it is much bluer than the isochrones' MS. Indeed it is located in the CMD region corresponding to the bulk of field stars (Sect. 4.1). The stars BT 1 and 13 are bluer than the isochrone giant branch, which indicates that they are not members. This is supported by their large parallaxes, locating them closer than what we find for NGC 1252. BT 11 is a probable member from its subgiant branch locus. Finally, BT 28 appears to be a probable member belonging to the TO region.

The present gain of $\Delta V \approx 2 \mathrm{mag}$ with respect to $\mathrm{BT}$ allows one to probe lower along the MS, which is a constraint for the TO, and isochrone fitting. Three additional GSC stars are present in the CCD images. According to the GSC, they are slightly fainter than those measured in Table 1. Assuming the additional stars as members their total number in the range $14<V<16$ would be 6 . Note that the isochrones (Fig. 5) have most probable members on the red MS side. This suggests them preferentially as part of a double star sequence, which is expected for a cluster remnant stellar content. Binary star sequences have been observed e.g. in the globular cluster 47 Tucanae (Santiago et al. 1996a) and the young LMC cluster NGC 1818 (Elson et al. 1998). Globular clusters appear to have a smaller binary fraction than open clusters. In the globular cluster NGC 2808 Ferraro et al. (1997) obtained a fraction of $24 \%$. For the post-core-collapse globular cluster NGC 6752 (Rubenstein \& Bailyn 1997) the overall fraction is low $(<16 \%)$ except in the inner core (15-38\%). González \& Lapasset (2000) estimated a frac-

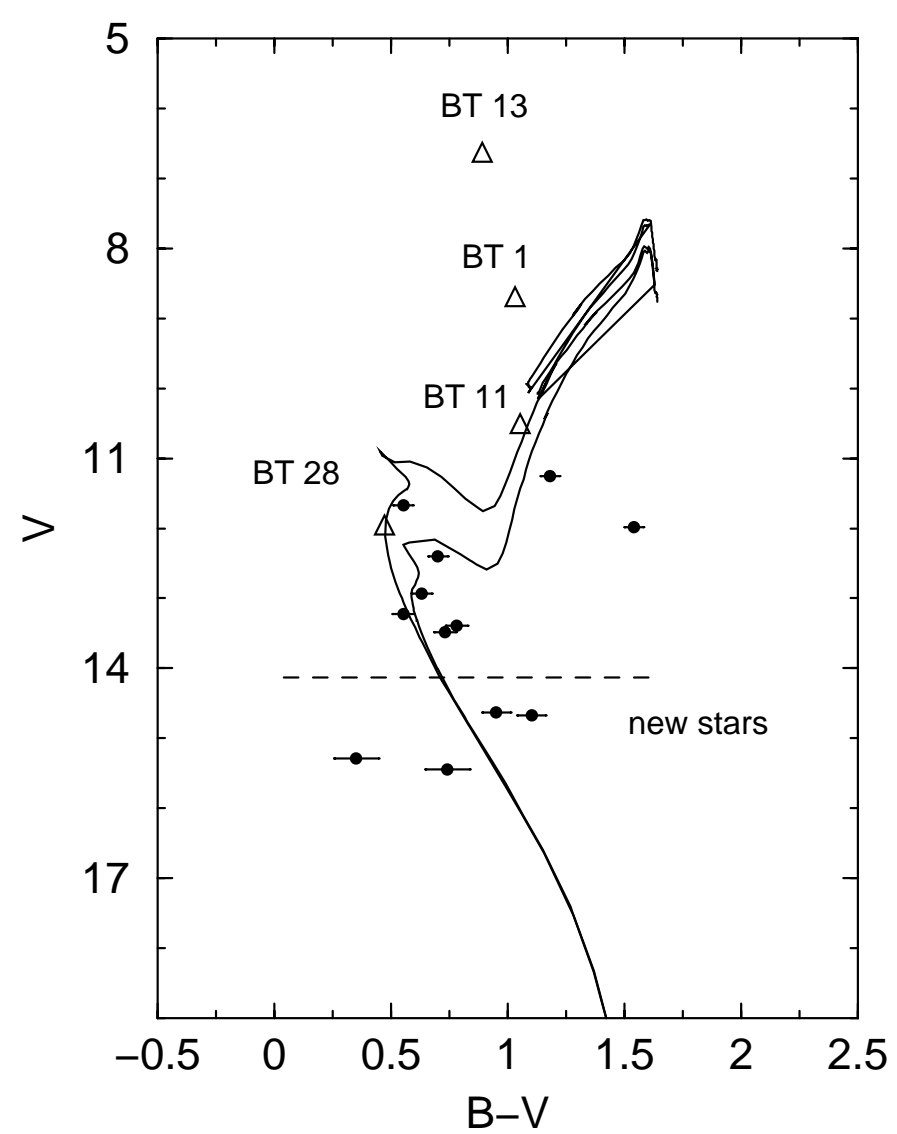

Fig. 5. NGC 1252: $V \times(B-V)$ diagram for 12 stars with CCD photometry (solid circles). Total errors in colours are shown. Open triangles are stars from Bouchet \& Thé's (1983) photometry. Line separates stars observed for the first time. Padova isochrone solutions are shown.

tion of $26 \%$ ( $46 \%$ including all suspected binaries) in the open cluster NGC 2516. From 167 bright Hyades members (Patience et al. 1998) the detected multiplicity is 59 binaries and 10 triples. This dynamically evolved open cluster has a detected binary fraction of $41 \%$. Binary fractions in globular clusters cannot be directly compared to those in open clusters, since loose binaries are thought to be destroyed at initially higher densities. Nevertheless in an advanced dynamical state for an open cluster or remnant one does not expect binaries to be destroyed by stellar interactions. Comparing the number of MS and TO stars on either side of the fitted isochrones for NGC 1252 (Fig. 5) a high binary fraction of $67 \%$ is obtained. The statistics is low, but taking this fraction at face value NGC 1252 would be consistent with a late dynamical state.

\subsection{Comparison with a field model}

We wish to compare the concentration CMD with that expected from field stars in the same direction and limiting magnitude, following Bassino et al. (2000). Bica et al. (2001) have compared integral star counts down to a limiting magnitude in their fields to those expected from a Galactic structure model. We use the same model as starting point to build a theoretical CMD of field stars in the 


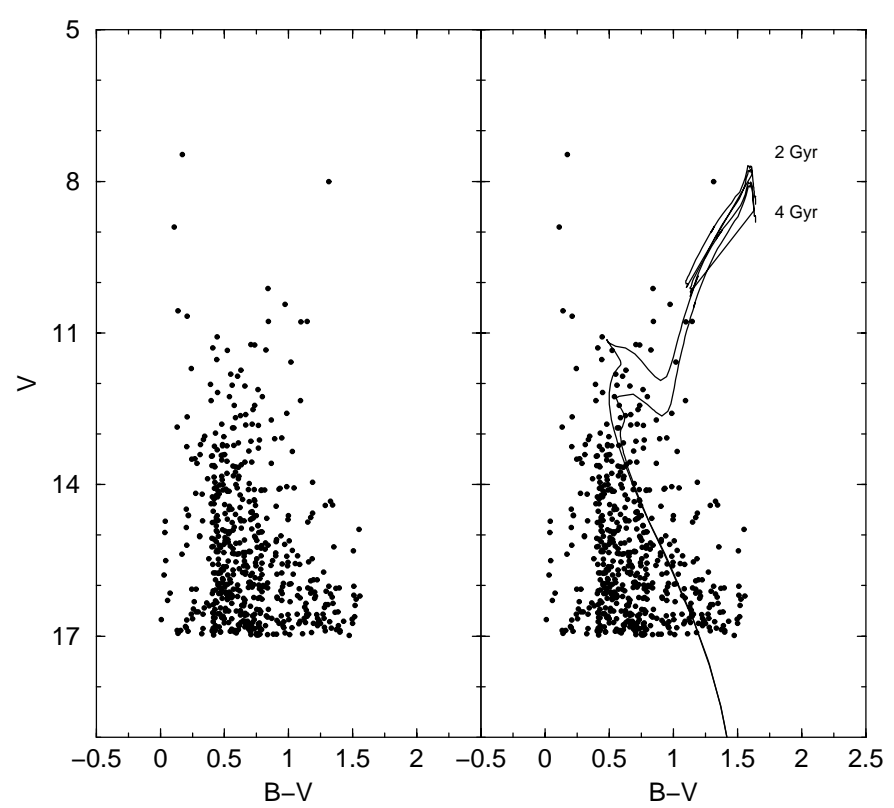

Fig. 6. Left panel: $V \times(B-V)$ diagram predicted by the Galactic structure model for a region of diameter $1^{\circ}$ in the same direction as NGC 1252. Right panel: same isochrone solution for NGC 1252 as in Fig. 5.

direction of NGC 1252. This model has been described in more detail by Reid \& Majewski (1993) and Santiago et al. (1996b). A recent application of the method to Galactic fields was carried out by Kerber et al. (2001). It includes 3 structural components: a thin disc, a thick disc and spheroid. The discs stellar density profiles are described by double exponentials, one along the plane of the disc and the other perpendicular to it. The stellar luminosity function is well constrained down to $M_{V} \approx 12$. Given the magnitude limit $(V \approx 15.5)$ for the concentration stars, our star counts are almost entirely dominated by the thin disc.

Given a direction, limiting magnitude and solid angle, the model provides expected numbers of stars throughout the CMD plane. In Fig. 6 we show a model CMD for a region of diameter $1^{\circ}$ around the concentration. This large area provides a statistically significant CMD. The isochrones on the right panel are from Fig. 5. They do not fit the bulk of field stars, which are considerably bluer in average. The isochrones are an upper envelope to the bulk of field stars and consequently NGC 1252 appears to be located in the field foreground. In Fig. 7 we present a similar CMD but for the small solid angle of the CCD data. This sample was randomly taken from the expected model counts in each CMD cell (scaled to the smaller solid angle) and allowing for Poisson fluctuations. As expected, a typical small field CMD will be populated preferentially with stars from the densest CMD zone in the large area (Fig. 6). However, although statiscally unlikely, one cannot rule out a fluctuation producing a CMD similar to that of the concentration. The model CMD (Fig. 6) extends down to $V \approx 17$, since unlike the CCD data limit (Fig. 7), it is not affected by incompleteness. In the par-

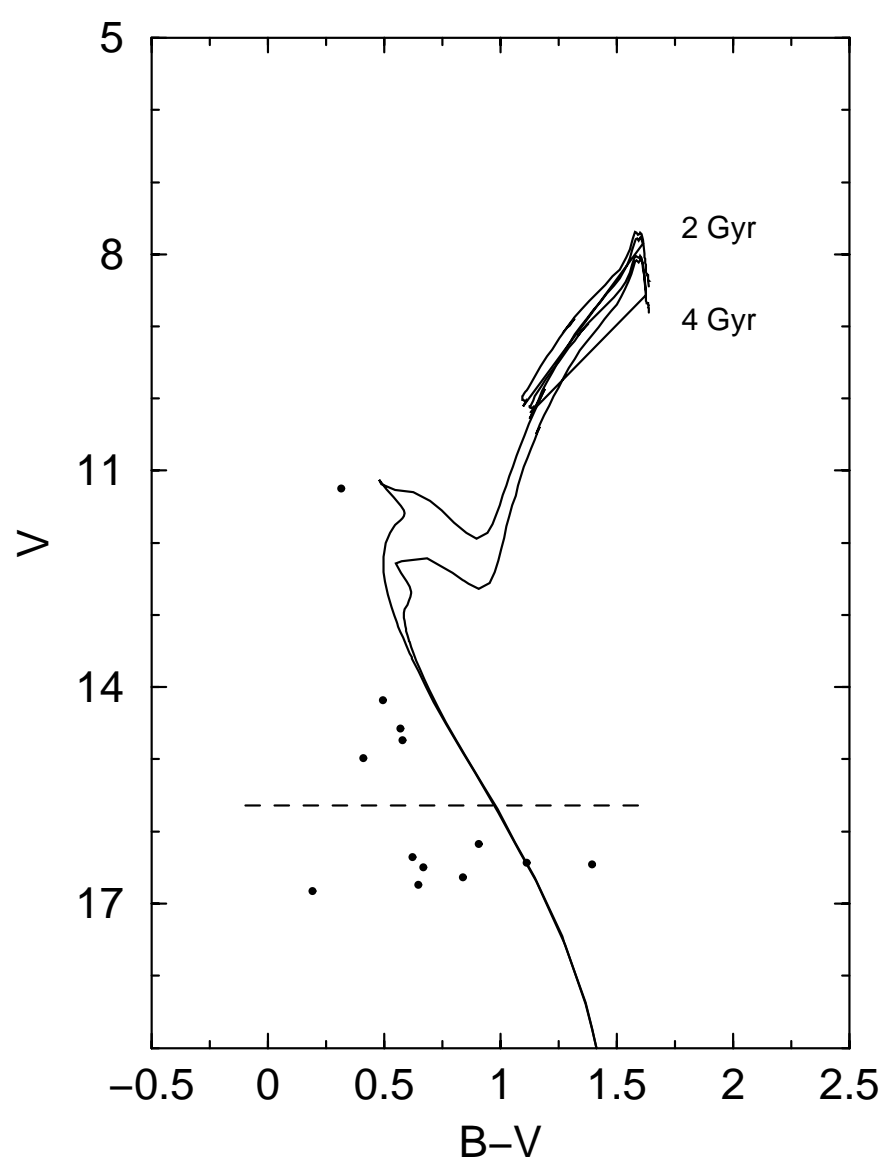

Fig. 7. Theoretical $V \times(B-V)$ Galactic field diagram for a solid angle equal to that of the CCD. Same isochrone solution for NGC 1252 (Fig. 5). Dashed line indicates the CCD limit.

ticular small field sampling the isochrones are located redwards of the artificial stars sampled. The NGC 1252 stars suggesting a MS (Fig. 5) are distributed in a region much narrower than the model stars (Fig. 6). These comparisons give support to the existence of a physical stellar group.

\subsection{Available astrometry}

We extracted Tycho stars within a $80^{\prime}$ diameter centered in the concentration. We show in Fig. 8 a sky chart with proper motions proportional to arrow sizes. The large circle has a diameter of $14^{\prime}$ and corresponds to the present stellar concentration. Note that stars fainter than $V \approx 12$ are absent in the Tycho catalogue. The bright giants BT 1 and 13 have different proper motions, in agreement with their different parallaxes. They are foreground field giants with respect to NGC 1252. The probable member BT 11 and the less probable one BT 15 (Table 1) present proper motions significantly different $\left(\mu_{\alpha}=+15.7 \pm 4.6 \mathrm{mas} / \mathrm{yr}\right.$, $\mu_{\delta}=+17.7 \pm 4.2 \mathrm{mas} / \mathrm{yr}$ and $\mu_{\alpha}=-0.4 \pm 5.4 \mathrm{mas} / \mathrm{yr}$, $\mu_{\delta}=+2.2 \pm 4.9$ mas $/ y r$, respectively), and they cannot be members simultaneously. Outside the concentration area (Fig. 8) three Hipparcos stars occur: HIP 14930 (TW Hor) at $d_{\odot}=403_{-74}^{+118} \mathrm{pc}, \mathrm{HIP} 15176$ at $d_{\odot}=397_{-149}^{+603} \mathrm{pc}$ and HIP 14975 at $d_{\odot}=630_{-210}^{+630}$ pc. From the distances and/or 


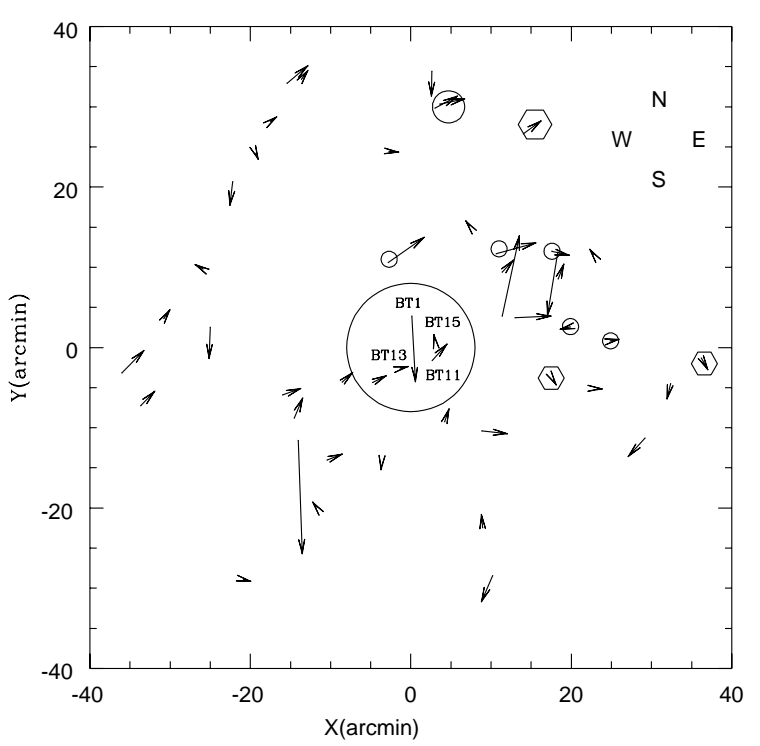

Fig. 8. NGC 1252: sky chart of Tycho stars in a region of diameter $80^{\prime}$ centered in the present concentration. Arrow length is proportional to proper motion modulus, the largest one is $121.2 \mathrm{mas} / \mathrm{yr}$. Large circle has a diameter of $14^{\prime}$ and encompasses NGC 1252. Hexagones are Hipparcos stars outside it. Small circles are stars with proper motions and photometry compatible within uncertainties with NGC 1252.

Johnson photometry in the Hipparcos catalogue, we concluded that they are non-members.

The available proper motions are not deep enough to study the TO and MS (Fig. 5). The faint stars $(12<V<$ $15.5)$ would be necessary for a conclusive proper motions result. A cautionary remark for proper motions and parallaxes based on observations spanning a few years, such as Tycho and Hipparcos data, is binarity. Early plates in the XX century like ACT's combined to subsequent images can lessen the problem, but not for binaries with periods longer than 100-200 years (Baumgardt 1998).

Based on $N$-body simulations Terlevich (1987) showed that a corona is expected after 300-400 Myr of evolution, and that such stars are likely to escape in the near future. We show in Fig. 9 the CMD of stars outside the concentration area with BT photometry. We distinguish BT's members and non-members. We superimpose the present isochrone solution (Fig. 5), which suggests that most of their non-members in the TO region are compatible with our interpretation of NGC 1252. A few giants considered by them to be members are as well possible members in our interpretation owing to their proximity to the isochrones. We conclude that 13 stars are compatible with the isochrones. Eight are marked in the Tycho chart (Fig. 8), since 2 are absent in the Tycho catalogue and 3 are outside the extraction. The marked stars are distributed on one side of the concentration because it is located at the edge of BT's large region. Six marked stars have proper motions compatible within uncertainties either with the probable member BT 11 or the less probable member BT 15, and they could be members of a corona, as seen in M 73 (Bassino et al. 2000). Tycho errors can be

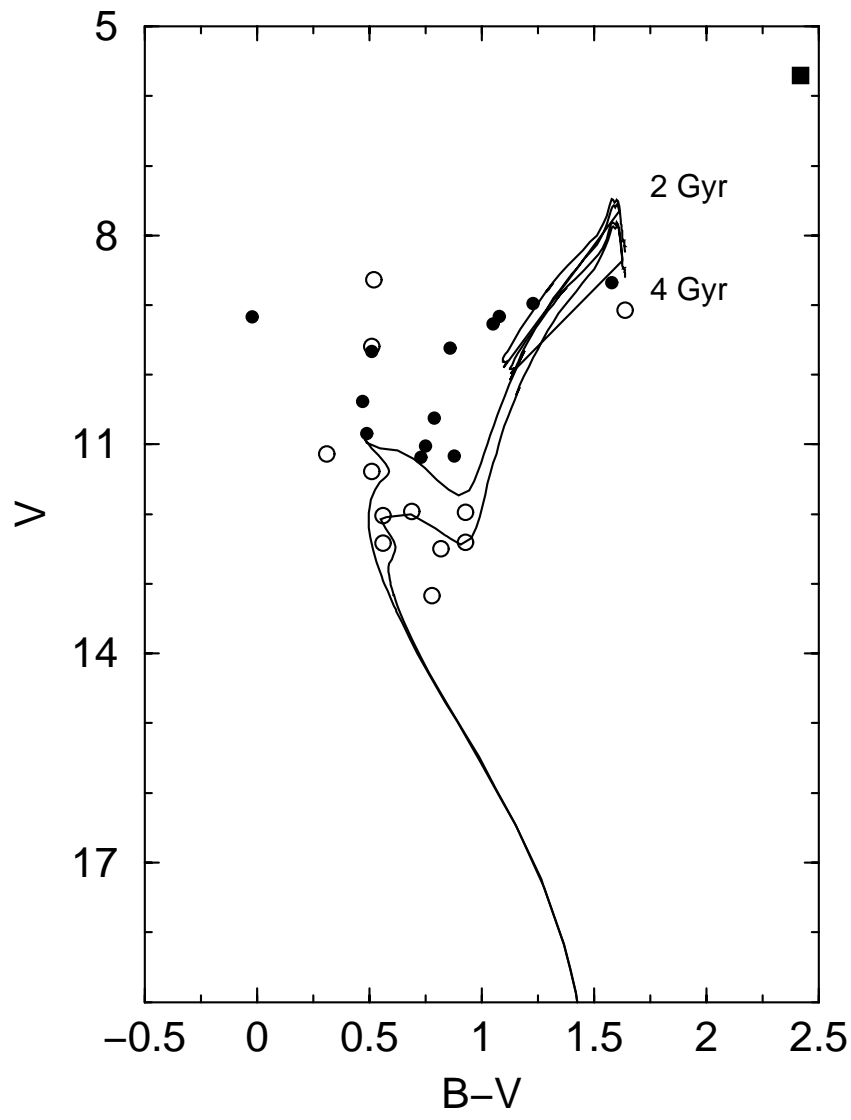

Fig. 9. $V \times(B-V)$ diagram for stars in BT's photometry located outside the concentration area. Solid circles are BT's member stars and open circles non-member stars according to their interpretation. Continuous lines: same NGC 1252 isochrone solution as in Fig. 5.

as large as 5 mas/yr for these stars, which at the object distance of $0.64 \mathrm{kpc}$ implies transversal velocity errors of $\approx 15 \mathrm{~km} \mathrm{~s}^{-1}$. Since velocity dispersions in open clusters are much lower (e.g. $0.23 \mathrm{~km} \mathrm{~s}^{-1}$ for the Hyades, Gunn et al. 1988) no inference can be made about internal motions in the present object.

\section{Comparisons with the Hyades}

The luminosity function ( $\mathrm{LF}$ ) can provide information on cluster mass and dynamical state. We take the Hyades as template to infer the properties of the present objects. We have selected and homogenized stars with $V<11$ (WEBDA), which encompass the available absolute mag intervals for NGC 1901 and NGC 1252. Adopting ( $m-$ $M)_{0}=3.32$ for the Hyades (WEBDA, Weidemann et al. 1992) we computed $M_{V}$ for corresponding giants, TO and MS stars which form the reference histograms in Figs. 10 and 11. For NGC 1901 and NGC 1252 we computed the $M_{V}$ histograms using the derived parameters and their 20 and 12 probable members, respectively (Sects. 3 and 4). We employed 181 Hyades members in the magnitude range of Fig. 10, and 147 in that of Fig. 11.

We show in Fig. 10 percentage fractions with respect to the total population of probable members in the 


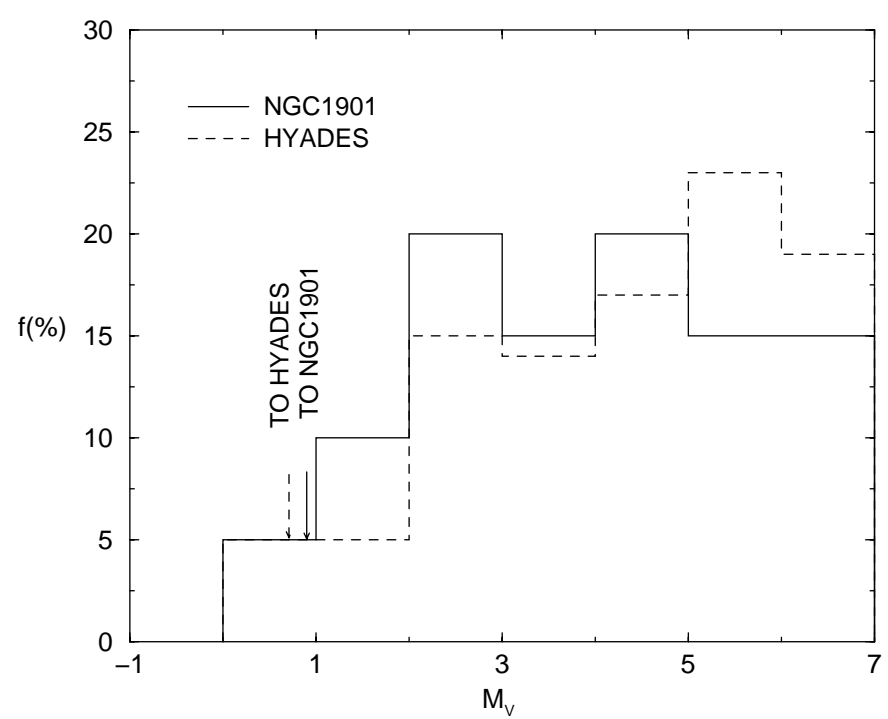

Fig. 10. Absolute $V$ luminosity function of NGC 1901 compared to that of the Hyades. Percentage fractions $f(\%)$ with respect to the total population of probable members in the magnitude range are shown.

available absolute mag range for NGC 1901, as compared to the Hyades. The histogram shapes are similar indicating a comparable mass function slope in the available range. CCD observations in the whole object area would improve the statistics in the present mag range, and deeper observations might reveal the behaviour of the low MS. The population ratio of stars in the two objects is $R_{\mathrm{P}}=9.1$, which suggests that the Hyades are this factor more massive than NGC 1901 as sampled by the CCD. However, SP members with proper motions from Murray et al. (1969) and the present Tycho analyses indicate 10 bright members in the CCD area and 10 outside it. This would indicate a mass ratiom $\mathcal{M}_{\text {Hyades }} / \mathcal{M}_{\text {NGC } 1901}=4.6$, assuming that both objects are in a similar dynamical state with equally populated lower MS.

In Fig. 11 we compare the LFs of NGC 1252 and the Hyades. The shapes are similar despite the limited population of stars for NGC 1252. In this case the object is essentially contained in the CCD so that $R_{\mathrm{P}}=12.3$, and the mass ratio is thus $\mathcal{M}_{\text {Hyades }} / \mathcal{M}_{\text {NGC } 1252}=12.3$, again under the assumption that both objects are in a similar dynamical state. It would be important to probe the lower MS $\left(M_{V}>6\right)$.

The Hyades have a nearly flat current mass function and appear to have lost $\approx 90 \%$ of an estimated total initial mass of $14000 M_{\odot}$ (Weidemann et al. 1992). In this sense the Hyades can be considered an open cluster remnant despite the fact that they are widely accepted as an open cluster. NGC 1901 and NGC 1252 have similar LFs as that of the Hyades in the available luminosity range, although they differ in terms of scaling factor. The inferred lower total masses, especially that of NGC 1252 for which evidence of a high binary fraction was found (Sect. 4), favour the concept of an open cluster remnant.

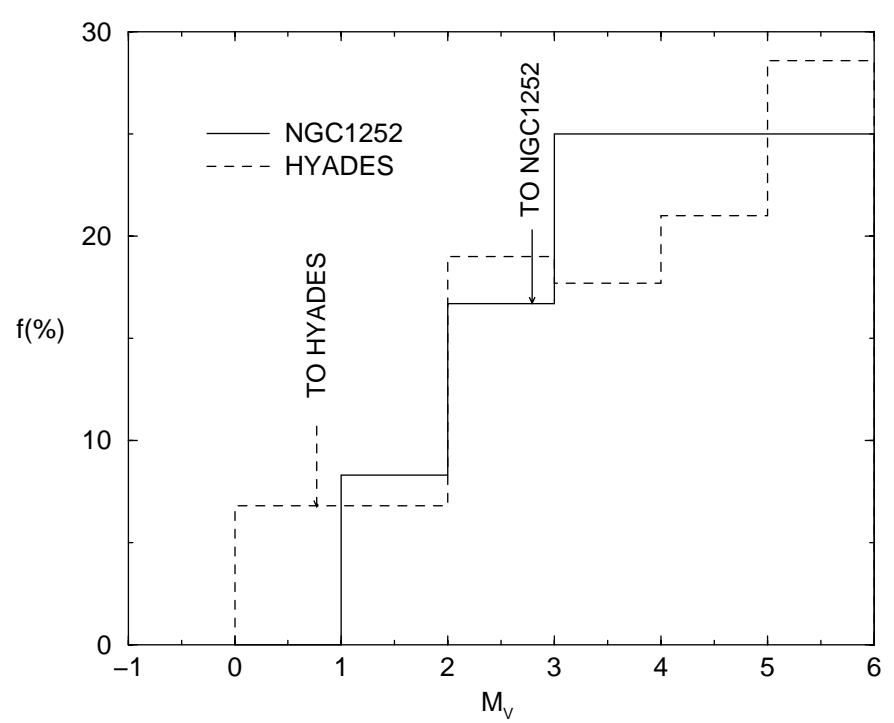

Fig. 11. Same as Fig. 10, for NGC 1252.

\section{Concluding remarks}

We provide CCD photometry of the stellar groups NGC 1901 and NGC 1252, probing them deeper than in the literature. We also explore the available Hipparcos and Tycho astrometries. The photometry confirms that NGC 1901 is a physical system which is also supported by proper motions. For NGC 1252 we describe a different set of stars as compared to the object interpreted by Bouchet \& Thé (1983), and questioned by Eggen (1984) and Baumgardt (1998). The present CMD suggests a TO and MS, so that a physical stellar group cannot be ruled out. The available astrometry is not helpful enough to constrain properties of this object.

NGC 1901 has an age comparable to that of the Hyades and their dynamical states are similar within the available LF range for NGC 1901. It would be important to further deepen NGC 1901's photometry to verify if depletion of lower MS stars occurs with respect to the Hyades, which would be the signature of a more evolved dynamical state. NGC 1252 is consistent with the notion of an old open cluster remnant, similar to M 73 (Bassino et al. 2000). NGC 1901 appear to a factor 4.6 less massive than the Hyades, while NGC 1252 is a factor 12.3, assuming equally populated lower main sequences.

In order to conclusively determine the nature and dynamical state of stellar groups like NGC1901 and NGC 1252 one needs deeper photometry and astrometry, and high dispersion spectroscopy for radial velocities and abundances. Binary fraction studies are fundamental too. Finally, from theoretical and numerical calculations it would be important to establish criteria to classify late stages of open cluster dynamical evolution and subsequent remnants. The inclusion of binaries and triples is crucial in the models. As a first step, the Hyades themselves 
require a clear determination of the dynamical state and classification as open cluster or open cluster remnant.

Acknowledgements. We thank an anonymous referee for important remarks, the students M. Campos and M. Aguero and the Bosque Alegre staff for assistance during the observations and hospitality, and Márcio R. de Oliveira (CNPq fellow) for discussions. We also thank Gerry Gilmore and Jim Lewis (IoA Cambridge) for the original software used for modeling the structure of the Galaxy. Part of the present data was extracted from the CDS database (Strasbourg). This work was partially supported by the Argentinian-Brazilian grant IRA 98/0967-9 by CONACyT/FAPERGS. We also acknowledge support from the Brazilian Institutions CNPq and CAPES.

\section{References}

Bassino, L. P., Waldhausen, S., \& Martinez, R. E. 2000, A\&A, 355,138

Bencivenni, D., Brocato, E., Buonanno, R., \& Castellani, V. 1991, AJ, 103, 137

Baumgardt, H. 1998, A\&A, 340, 402

Bica, E., Santiago, B. X., Dutra, C. M., et al. 2001, A\&A, 366, 827

Bica, E., Geisler, D., Dottori, H., et al. 1998, AJ, 116, 723

Bica, E., Schmitt, H. R., Dutra, C. M., \& Luz Oliveira, H. 1999, AJ, 117, 238

Bouchet, R., \& Thé, P. S. 1983, PASP, 95, 474

Burstein, D., \& Heiles, C. 1982, AJ, 87, 1165

Carraro, G., \& Patat, F. 1995, MNRAS, 276, 563

Carraro, G. 2000, A\&A, 357, 145

Combes, F., Leon, S., \& Meylan, G. 1999 A\&A, 352, 149

de la Fuente Marcos, R. 1998, A\&A, 333, L27

Dolphin, A. E., \& Hunter, D. A. 1998, AJ, 116, 1275

Eggen, O. J. 1984, PASP, 96, 70

Elson, R., Sigurdsson, S., Davies, M., Hurley, J., \& Gilmore, G. 1998, MNRAS, 300, 857

Ferraro, F. R., Carretta, E., Fusi Pecci, F., \& Zamboni, A. 1997, A\&A, 327, 598
Friel, E. D. 1995, ARA\&A, 33, 381

Girardi, L., Bressan, A., Bertelli, G., \& Chiosi, C. 2000, A\&AS, 141,371

González, J. F., \& Lapasset, E. 2000, AJ, 119, 2296

Gunn, J. E., Griffin, R. F., Griffin, R. E. M., \& Zimmerman, B. A. 1988 , AJ, 96,198

Hassan, S. M. 1974, A\&AS, 20, 255

Kerber, L., Javiel, S., \& Santiago, B. X. 2001, A\&A, 365, 424

Lodén, L. O. 1977, A\&AS, 29, 31

Mermilliod, J. C. 1996, in The origins, evolution, and destinies of binary stars in clusters, ASP Conf. Ser., 90, 475

Minniti, D., Clariá, J. J., \& Gomez, M. N. 1989, Ap\&SS, 158,9

Monet, D., Bird, A., Canzian, B., et al. 1998, USNO-A2.0 Catalogue, CDS database, Strasbourg

Murray, C. A., Dickens, R. J., \& Walker, E. N. 1969, Obs., 89, 104

Patience, J., Ghez, A. M., Reid, I. N., Weinberger, A. J., \& Matthews, K. 1998, AJ, 115, 1972

Reid, I. M. 1993, ARA\&A, 31, 345

Reid, I. M., \& Majewski, S. R. 1993, ApJ, 409, 635

Rubenstein, E. P., \& Bailyn, C. D. 1997, ApJ, 474, 701

Sanduleak, N., \& Philip, A. G. D. 1968, AJ, 73, 566

Santiago, B. X., Elson, R., \& Gilmore, G. 1996a, MNRAS, 281, 1363

Santiago, B. X., Gilmore, G., \& Elson, R. 1996b, MNRAS, 281, 871

Schlegel, D. J., Finkbeiner, D. P., \& Davis, M. 1998, ApJ, 500, 525

Stetson, P. B. 1992, in IAU Colloq. 136, Stellar Photometry Current Techniques and Future Developments, ed. C. J. Butler, \& Elliott (Cambridge Univ. Press), 291

Sulentic, J. W., \& Tifft, W. G. 1973, in The Revised New General Catalogue of Nonstellar Astronomical Objects (The University of Arizona press, Tucson)

Terlevich, E. 1987, MNRAS, 224,193

Tucholke, H.-J., de Boer, K. S., \& Seitter, W. C. 1996, A\&AS, 119, 91 (MACS Catalogue)

Weidemann, V., Jordan, S., Iben, I. Jr., \& Casertano, S. 1992, AJ, 104, 1876

Wielen, R. 1971, A\&A, 13, 309 